\title{
Controlled vanadium doping of mos2 thin films through co-sputtering and thermal sulfurization
}

\author{
Cihan KURU ${ }^{1,2 *}$ (iD) \\ ${ }^{1}$ Department of Metallurgical and Materials Engineering, Bilecik Seyh Edebali University, Bilecik, 11230, Turkey \\ ${ }^{2}$ Biotechnology Application and Research Centre, Bilecik Seyh Edebali University, Bilecik, 11230, Turkey
}

\begin{abstract}
Recently, transition metal dichalcogenides (TMDs) have gained great attention owing to their remarkable properties. The electronic structure of TMDs can be modified by substitutional doping, which could give rise to novel and exciting properties. In this study, a strategy is presented for controlled vanadium (V) doping of $\mathrm{MoS}_{2}$, in which $\mathrm{V}$ doped $\mathrm{MoS}_{2}$ films with good uniformity are prepared by thermal sulfurization of V-Mo alloy films deposited using co-sputtering. The $\mathrm{V}$ incorporation in $\mathrm{MoS}_{2}$ induces $\mathrm{p}$ type doping, which enhances the electrical conductivity of $\mathrm{MoS}_{2}$ by a factor of 35-40. Such doping strategy and consequent conductivity improvement may be useful in many applications such as catalysis, nanoelectronics and optoelectronics.
\end{abstract}

\section{Article info \\ History:}

Received:07.08.2019

Accepted:11.03.2020

Keywords:

$\mathrm{MoS}_{2}$,

doping,

vanadium,

transition metal

dichalcogenides,

thin film

\section{Introduction}

Since the discovery of graphene, there has been a growing interest in 2D materials [1-4]. Analogous to graphene, TMDs exhibit a layered structure, where each layer has a composition of $\mathrm{MX}_{2}(\mathrm{M}$ : transition metal, X: S, Se or Te) [5]. The transition metal and chalcogenide atoms are linked by strong covalent bonds whereas individual layers are stacked together by weak van der Waals interactions [5]. TMDs have been extensively studied for their electronic, optoelectronic, sensing, catalytic and tribological properties [6-10]. One of the most widely investigated TMD material is $\mathrm{MoS}_{2}$. Bulk $\mathrm{MoS}_{2}$ is an indirect bandgap semiconductor with a gap value of $1.2 \mathrm{eV}$ while single layer $\mathrm{MoS}_{2}$ has a direct bandgap of $1.8 \mathrm{eV}$ [11]. Field effect transistors fabricated using single layer $\mathrm{MoS}_{2}$ show high on/off current ratios with decent carrier mobility, which makes it promising for low power and flexible electronics $[12,13]$. Ultrasensitive photodetectors have been fabricated using $\mathrm{MoS}_{2}$ $[14,15]$. Low friction $\mathrm{MoS}_{2}$ lubricant coatings have been demonstrated [16,17]. $\mathrm{MoS}_{2}$ has also been shown to be a promising catalyst for hydrogen evolution reaction (HER) [18-22].

Doping has been used in semiconductor industry for many decades to improve device performance. Similarly, doping can be utilized to alter the electronic structure of $\mathrm{MoS}_{2}$ opening new venues in electronics, optoelectronics and catalysis. For example, Se doped $\mathrm{MoS}_{2}$ nanosheets with tunable optical properties have been synthesized by simultaneous sulfurization and selenization of $\mathrm{MoO}_{3}$ powder, in which Se doping causes a shift in the bandgap value of $\mathrm{MoS}_{2}$ [23]. Nipane $e t$ al. demonstrated p-type doping of $\mathrm{MoS}_{2}$ by low energy phosphorus implantation [24]. Lateral p-n junction devices fabricated by selective regional phosphorus doping of $\mathrm{MoS}_{2}$ exhibited nearly ideal and air stable diode behavior. Furthermore, chlorine doping was shown to reduce the contact resistance of $\mathrm{MoS}_{2}$ by 2-3 orders of magnitude, resulting in enhanced mobility [25]. Moreover, Pulickel et al. [26], Xie et al. [27] and Li et al. [28] investigated the electronic transport properties of $\mathrm{W}_{\mathrm{x}} \mathrm{Mo}_{1-\mathrm{x}} \mathrm{S}_{2}$, $\mathrm{MoS}_{2 \mathrm{x}} \mathrm{Se}_{2(1-\mathrm{x})}$ and $\mathrm{Co}_{\mathrm{x}} \mathrm{Mo}_{1-\mathrm{x}} \mathrm{S}_{2}$ alloys.

The catalytic activity of $\mathrm{MoS}_{2}$ is hindered by low conductivity and lack of active sites on its basal plane [21]. Theoretical studies suggest that substitutional doping of $\mathrm{MoS}_{2}$ can increase the conductivity as well as alter the binding energy of adsorbed hydrogen on sulfur favorably, inducing additional active sites, which would in turn enhance HER activity [29,30]. In the light of these potential benefits, many doping strategies for $\mathrm{MoS}_{2}$ have been developed. Solution processing methods have been used to synthesize $\mathrm{Zn}$, $\mathrm{Ni}, \mathrm{Co}, \mathrm{Fe}, \mathrm{Cu}$ doped $\mathrm{MoS}_{2}$ nanosheets [31-33]. P, Se 
and $\mathrm{V}$ doped $\mathrm{MoS}_{2}$ prepared by high temperature solid state reaction techniques have been reported [34-36]. In addition, $\mathrm{Cr}$ doped $\mathrm{MoS}_{2}$ films and $\mathrm{MoS}_{2(1-x)} \mathrm{Se}_{2 x}$ nanobelts have been produced by CVD method [37,38].

In this study, a method is reported for controllable $\mathrm{V}$ doping of $\mathrm{MoS}_{2}$. V doped $\mathrm{MoS}_{2}$ thin films with different V/Mo ratios were prepared through cosputtering of V-Mo alloy films and subsequent thermal sulfurization step. Simultaneous deposition of $\mathrm{V}$ and Mo atoms by co-sputtering enables to synthesize homogeneously doped $\mathrm{MoS}_{2}$ films with good control of dopant concentration and thickness. The structural properties of the $\mathrm{V}$ doped $\mathrm{MoS}_{2}$ films were characterized by X-ray diffraction (XRD), Raman spectroscopy, scanning electron microscopy (SEM) and energy dispersive X-ray spectroscopy (EDX) measurements. Four-point probe measurements were conducted to reveal the effect of $\mathrm{V}$ doping on the sheet resistance of $\mathrm{MoS}_{2}$ film.

\section{Materials and Methods}

A multi-target magnetron sputtering system (Vaksis Angora) was used for the deposition of the V-Mo alloys. $20 \mathrm{~nm}$ thick V-Mo alloy films were deposited on n-type (100) Si substrates by co-sputtering of V and Mo targets (99.9\% purity, Kurt Lesker) in RF and DC mode, respectively. The chamber was evacuated to $5 \times 10^{-6}$ Torr before deposition. The Ar pressure was kept at 10 mTorr during the sputtering. Targets were cleaned at each run by pre-sputtering for 5 minutes while shutters were closed. The substrate holder was rotated at $5 \mathrm{rpm}$ during the deposition to ensure uniformity. The desired alloy content was obtained by varying the sputter rate of each target, which was controlled by sputter power. Four different samples of $\mathrm{V}_{\mathrm{x}} \mathrm{Mo}_{1-\mathrm{x}} \mathrm{S}_{2}$ were prepared with increasing amounts of $\mathrm{X}$.

The sulfurization was carried out in a tube furnace (MTI OTF-1200X-S-NT-LD) with $50.8 \mathrm{~mm}$ diameter quartz tube. The deposited alloys films were placed at the center and $0.5 \mathrm{~g}$ sulfur powder (Merck) at the upstream side of the furnace. The tube was flashed with high purity nitrogen to remove oxygen for $1 \mathrm{~h}$ prior to sulfurization and nitrogen flow with a rate of $100 \mathrm{sccm}$ was continued during the sulfurization. The center of the furnace was gradually heated up to $800{ }^{\circ} \mathrm{C}$ in 80 min and kept at $800{ }^{\circ} \mathrm{C}$ for $1 \mathrm{~h}$ before cooled down to room temperature naturally. The sulfur powder was at $150{ }^{\circ} \mathrm{C}$ (above its melting temperature) during the reaction.
X-ray diffraction measurements were carried out by Rigaku D-Max with $\mathrm{Cu} \mathrm{Ka}$ source. The morphology and chemical composition of the films were investigated with Zeiss Supra 40VP scanning electron microscope. Raman spectroscopy measurements were performed on Kaiser Raman Rxn with a laser wavelength of $514 \mathrm{~nm}$. The sheet resistance of the films was determined by four-point probe measurements.

\section{Results and Discussions}

XRD measurements were performed in order to examine the crystal structure of the $\mathrm{V}$ doped $\mathrm{MoS}_{2}$ films (Figure 1). The XRD patterns of all samples are in good agreement with hexagonal $\mathrm{MoS}_{2}$ phase (PDF\#01-075-1539). The fact that no $\mathrm{VS}_{2}$ phase was detected and all samples exhibit hexagonal $\mathrm{MoS}_{2}$ phase indicate that $\mathrm{V}$ substitutionally doped $\mathrm{MoS}_{2}$ and the crystal structure of $\mathrm{MoS}_{2}$ has been preserved. The undoped $\mathrm{MoS}_{2}$ film exhibits two pronounced peaks originated from (100) and (101) planes due to the preferential growth of the film in vertical direction [39]. With the increasing V content, the (002) peak becomes prominent whereas the intensity of (100) and (101) peaks decreases. The intensity ratio of the (002) peak to the (100) peak is calculated to be $0.19,2.24$, 2.59, 5.44 and 25.4 for the $\mathrm{MoS}_{2}, \mathrm{~V}_{0.09} \mathrm{Mo}_{0.91} \mathrm{~S}_{2}$, $\mathrm{V}_{0.23} \mathrm{Mo}_{0.77} \mathrm{~S}_{2}, \mathrm{~V}_{0.33} \mathrm{Mo}_{0.67} \mathrm{~S}_{2}$ and $\mathrm{V}_{0.5} \mathrm{Mo}_{0.5} \mathrm{~S}_{2}$ films, respectively. The increasing ratio of (002) to (100) with higher $\mathrm{V}$ concentration indicates that $\mathrm{V}$ doping promotes the horizontal stacking. Furthermore, the interplanar spacing of the doped $\mathrm{MoS}_{2}$ films is close to that of the undoped $\mathrm{MoS}_{2}$ film, further suggesting that $\mathrm{V}$ doping did not alter the crystal structure of the $\mathrm{MoS}_{2}$ film significantly.

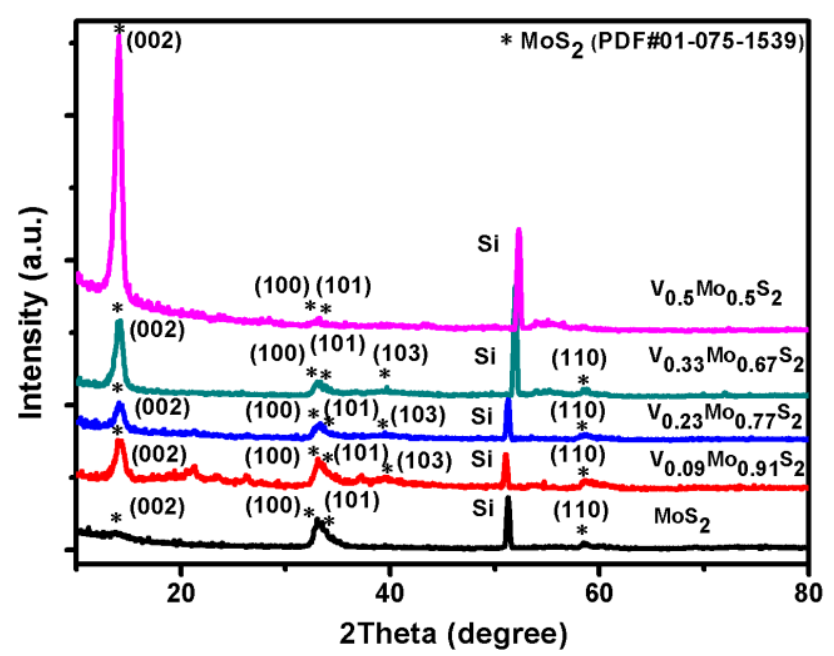

Fig. 1. XRD plots of the undoped $\mathrm{MoS}_{2}$ and V doped $\mathrm{MoS}_{2}$ films. 
The Raman spectra of the undoped $\mathrm{MoS}_{2}$ and $\mathrm{V}$ doped $\mathrm{MoS}_{2}$ films are shown in Figure 2. All the samples exhibit the characteristic peaks of $\mathrm{MoS}_{2}: \mathrm{E}_{2 \mathrm{~g}}^{1}$ originated from the in-plane vibrations of Mo-S atoms and $\mathrm{A}_{1 \mathrm{~g}}$ resulted from the out-of-plane vibrations of Mo-S atoms [40]. Note that both peaks are significantly broadened compared to atomically thin $\mathrm{MoS}_{2}$ films reported in literature [41, 42], which may be resulted from the polycrystalline nature of the films. No peaks corresponding to $\mathrm{VS}_{2}$ has been detected, confirming the substitutional doping of $\mathrm{V}$ in $\mathrm{MoS}_{2}$. The undoped $\mathrm{MoS}_{2}$ film shows an ambiguous $\mathrm{E}_{2 \mathrm{~g}}^{1}$ peak and a strong $A_{1 g}$ peak pointing out the vertically grown $\mathrm{MoS}_{2}$ layers [43]. However, as the $\mathrm{V}$ concentration is increased, the intensity ratio of $E_{2 g}^{1}$ to $A_{1 g}$ grows dramatically, which implies a transition from vertical to horizontal growth with increasing V/Mo ratio. These results are also consistent with the XRD outcome.

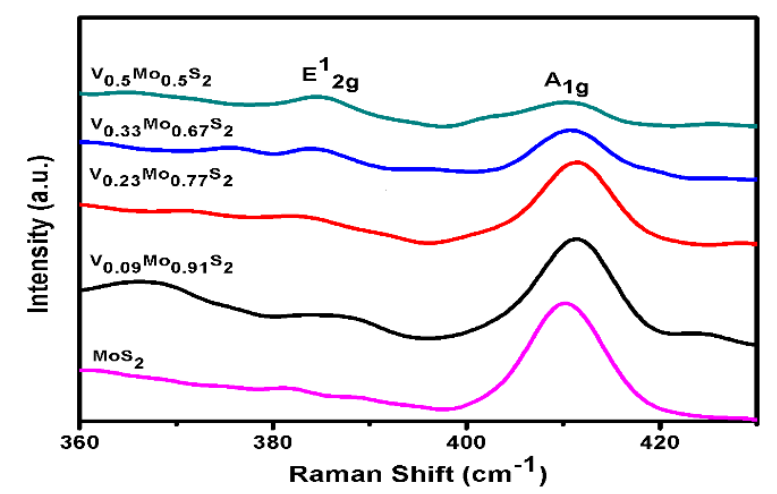

Fig. 2. Raman spectra of the undoped $\mathrm{MoS}_{2}$ and $\mathrm{V}$ doped $\mathrm{MoS}_{2}$ films.

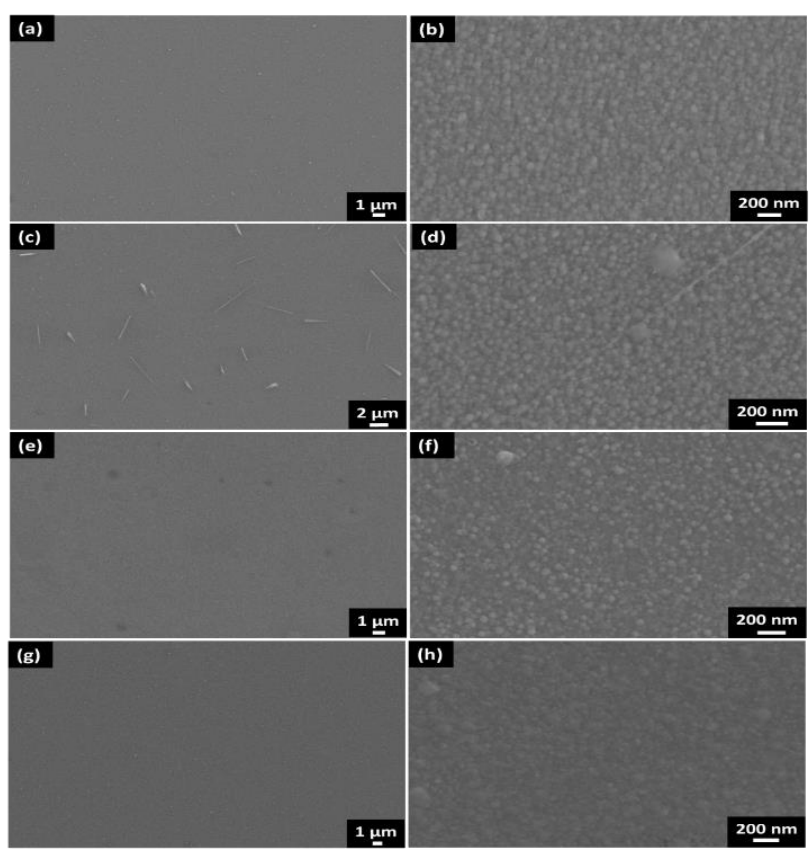

Fig. 3. Scanning electron microscopy images of $(a, b)$ $\mathrm{V}_{0.09} \mathrm{Mo}_{0.91} \mathrm{~S}_{2}$, (c, d) $\mathrm{V}_{0.23} \mathrm{Mo}_{0.77} \mathrm{~S}_{2}$, (e, f) $\mathrm{V}_{0.33} \mathrm{Mo}_{0.67} \mathrm{~S}_{2}$ and (g, h) $\mathrm{V}_{0.5} \mathrm{Mo}_{0.5} \mathrm{~S}_{2}$ films.
Figure 3 shows the SEM images of $20 \mathrm{~nm}$ thick V-Mo alloys sulfurized at $800{ }^{\circ} \mathrm{C}$ for $1 \mathrm{~h}$, in which $\mathrm{V}_{0.09} \mathrm{Mo}_{0.91} \mathrm{~S}_{2}, \mathrm{~V}_{0.23} \mathrm{Mo}_{0.77} \mathrm{~S}_{2}$ and $\mathrm{V}_{0.33} \mathrm{Mo}_{0.67} \mathrm{~S}_{2}$ have a granular morphology (50 $\mathrm{nm}$ average grain size) while $\mathrm{V}_{0.5} \mathrm{Mo}_{0.5} \mathrm{~S}_{2}$ exhibits more like a layered structure. Additionally, nanoribbon-like structures with widths down to $20 \mathrm{~nm}$ and lengths up to $6 \mu \mathrm{m}$ were detected in $\mathrm{V}_{0.09} \mathrm{Mo}_{0.91} \mathrm{~S}_{2}$ and $\mathrm{V}_{0.23} \mathrm{Mo}_{0.77} \mathrm{~S}_{2}$ films (Figure $3 \mathrm{a}$, c and d). The EDX mapping results confirm the presence of $\mathrm{S}, \mathrm{V}$ and Mo elements and indicate that their distribution is homogeneous throughout the films (Figure 4).

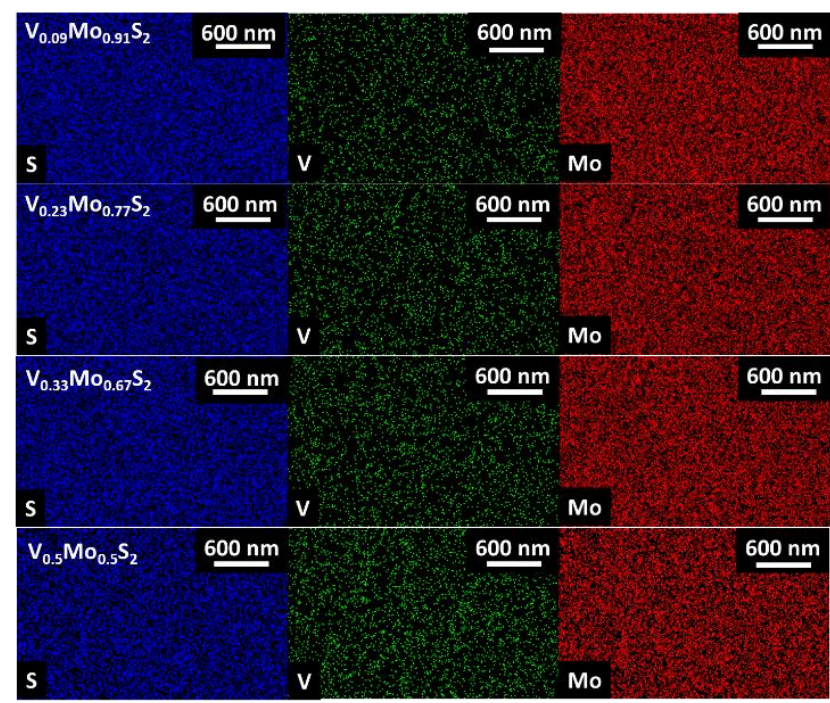

Fig. 4. EDX mapping results of the V doped $\mathrm{MoS}_{2}$ films.

Four-point probe measurements were conducted in order to reveal the effect of $\mathrm{V}$ doping on the electrical characteristics of $\mathrm{MoS}_{2}$. Figure 5 shows the sheet resistance values of the undoped and doped films, in which the doped films exhibit 35-40 times lower sheet resistance compared to undoped $\mathrm{MoS}_{2}$. The lowest sheet resistance value of $1.96 \mathrm{kohm} / \mathrm{sq}$ has been achieved at 33 at. $\% \mathrm{~V}$ doping. As $R_{s}=\rho . t$ and $\sigma=$ $1 / \rho\left(R_{S}\right.$ sheet resistance, $\rho$ resistivity, $t$ thickness and $\sigma$ conductivity), it can be concluded that $\mathrm{V}$ doping has increased the conductivity of the $\mathrm{MoS}_{2}$ film by a factor of 35-40. The conductivity of a semiconductor is given by $\sigma=q\left(n \mu_{e}+p \mu_{h}\right)$, where $q$ is the elemental charge, $n$ and $p$ are the electron and hole carrier concentration, $\mu_{e}$ and $\mu_{h}$ are the electron and hole mobility. Thus, the conductivity enhancement in the $\mathrm{V}$ doped $\mathrm{MoS}_{2}$ films can be attributed to the increase of carrier concentration. Since V has one less valance electron than Mo, the incorporation of $\mathrm{V}$ atoms in the $\mathrm{MoS}_{2}$ crystal causes an electron deficiency in the bonding orbitals; hence p-type doping is induced in $\mathrm{MoS}_{2}$. 


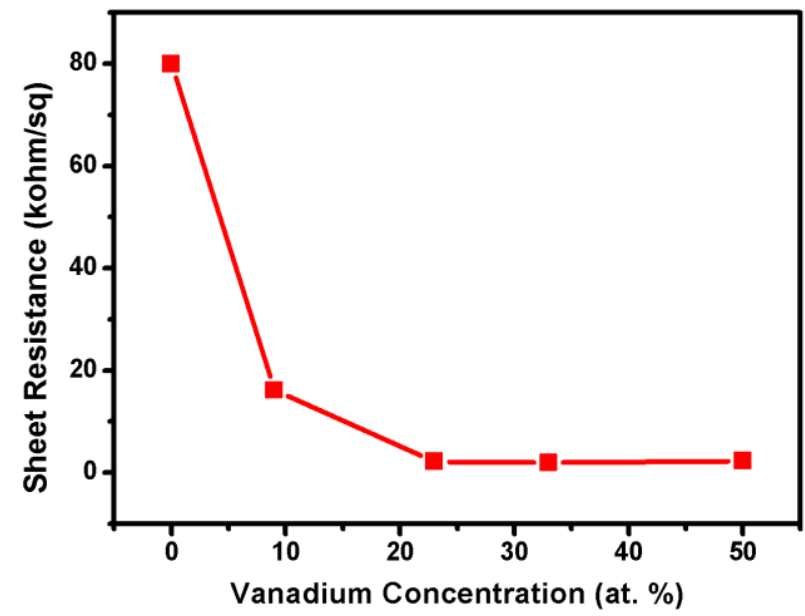

Fig. 5. The effect of $\mathrm{V}$ dopant concentration on the sheet resistance of the $\mathrm{MoS}_{2}$ film.

\section{Conclusions}

In summary, $\mathrm{V}$ doped $\mathrm{MoS}_{2}$ films with varying V/Mo ratios have been successfully prepared by sulfurization of sputter deposited V-Mo alloys. The doping concentration is adjusted by changing the relative deposition rates of $\mathrm{V}$ and Mo. The structural characterization results show that $\mathrm{MoS}_{2}$ hexagonal structure is maintained upon $\mathrm{V}$ doping, $\mathrm{V}$ doping is uniform throughout the films and horizontal layer stacking becomes preferred with increasing $\mathrm{V}$ content. The $\mathrm{V}$ doped $\mathrm{MoS}_{2}$ films exhibit greatly enhanced conductivity, which could be useful for catalysis applications such as hydrogen evolution or oxygen reduction reactions. Moreover, this method can be extended to synthesize various ternary TMD alloys, where controlled doping, thickness and morphology may be beneficial to study fundamental properties of such alloyed materials.

\section{Acknowledgements}

This work was supported by The Scientific and Technological Research Council of Turkey (Grand No. 117M257). I would like to thank Dr. Zeliha Ertekin for her help with the four-point probe measurements.

\section{References}

[1] Mas-Ballesté R., Gómez-Navarro C., GómezHerrero J. and Zamora F. 2D materials: To graphene and beyond. Nanoscale, 3-1 (2011) 20-30.

[2] Lin T.W., Chang C.S., Chang K.D., Zhang W., Yu Y.C., Zhang X.Q., Chang M.T., Li L.J., Wang J.T.W, Lee Y.H. and Lin C.T. Synthesis of Large-Area MoS2 Atomic Layers with Chemical Vapor Deposition. Adv. Mater., 24-17 (2012) 2320-2325.

[3] Gomathi A., Late D.J., Pati S.K., Manna A.K., Rao C.N.R., Ramakrishna Matte H.S.S. and Datta R. MoS2 and WS2 Analogues of Graphene, Angew. Chemie Int. Ed., 49-24 (2010) 4059-4062.

[4] Hayashi T., Terrones H., Pradhan N.R., CastroBeltrán A., Long A.D., Mallouk T.E., Endo M., Gutiérrez H.R., Feng S., Kim Y.A., Lv R., Perea-López N., Balicas L., Elías A.L., LópezUrías F., Berkdemir A. and Terrones M. Controlled Synthesis and Transfer of LargeArea WS2 Sheets: From Single Layer to Few Layers , ACS Nano, 7-6 (2013) 5235-5242.

[5] Li L.J., Eda G., Zhang H., Loh K.P., Shin H.S. and Chhowalla $M$. The chemistry of twodimensional layered transition metal dichalcogenide nanosheets. Nat. Chem., 5-4 (2013) 263-275.

[6] Strano M.S., Kis A., Coleman J.N., Wang Q.H. and Kalantar-Zadeh K. Electronics and optoelectronics of two-dimensional transition metal dichalcogenides. Nat. Nanotechnol., 7-11 (2012) 699-712.

[7] Baugher B.W.H., Churchill H.O.H., Yang Y. and Jarillo-Herrero P. Intrinsic electronic transport properties of high-quality monolayer and bilayer MoS2. Nano Lett., 13-9 (2013) 4212-4216.

[8] Li B.L., Wang J., Zou H.L., Garaj S., Lim C.T., Xie J., Li N.B. and Leong D.T. LowDimensional Transition Metal Dichalcogenide Nanostructures Based Sensors. Adv. Funct. Mater., 26-39 (2016) 7034-7056.

[9] Wang H., Lu Z., Xu S., Kong D., Cha J.J., Zheng G., Hsu P.C., Yan K., Bradshaw D., Prinz F.B. and Cui Y. Electrochemical tuning of vertically aligned MoS2 nanofilms and its application in improving hydrogen evolution reaction. Proc. Natl. Acad. Sci. U.S.A., 110-49 (2013) 1970119706.

[10] Simmonds M.C., Savan A., Pflüger E. and Van Swygenhoven H. Mechanical and tribological performance of MoS2 co-sputtered composites. Surf. Coatings Technol., 126-1 (2000) 15-24.

[11] Giacometti V., Radisavljevic B., Radenovic A., Brivio J. and Kis A. Single-layer MoS2 transistors. Nat. Nanotechnol., 6-3 (2011) 147150.

[12] Pu J., Yomogida Y., Liu K., Li L., Iwasa Y. and Takenobu T. Highly flexible MoS2 thin-film transistors with ion gel dielectrics. Nano Lett., 12-8 (2012) 4013-4017. 
[13] Yoon Y., Ganapathi K. and Salahuddin S. How Good Can Monolayer MoS2 Transistors Be ?. Electr. Eng., 11-9 (2011) 3768-3773.

[14] Meng X., Tang M., Liao L., Chu J., Hu W., Wang X., Lin T., Wang J., Sun S., Shen H., Zhou X., Huang H., Chen X., Jiang A., Wang P., $\mathrm{Lu} \mathrm{W.,} \mathrm{Sun} \mathrm{J.} \mathrm{and} \mathrm{Guo} \mathrm{N.} \mathrm{Ultrasensitive} \mathrm{and}$ Broadband MoS2 Photodetector Driven by Ferroelectrics. Adv. Mater., 27-42 (2015) 65756581.

[15] Kufer D. and Konstantatos G. Highly Sensitive, Encapsulated MoS2 Photodetector with Gate Controllable Gain and Speed. Nano Lett. 15-11 (2015) 7307-7313.

[16] Renevier N.M., Hamphire J., Fox V.C., Witts J., Allen T. and Teer D.G. Advantages of using self-lubricating, hard, wear-resistant MoS2based coatings. Surf. Coatings Technol., 142 (2001) 67-77.

[17] Donnet C., Le Mogne T., Martin J.M., Fayeulle S., Tonck A., Moncoffre N. and Millard-Pinard N. Nature of super-lubricating MoS2 physical vapor deposition coatings. J. Vac. Sci. Technol. A Vacuum, Surfaces, Film. 12-4 (2002) 19982004.

[18] Hinnemann B., Moses P.G., Bonde J., Jørgensen K.P., Nielsen J.H., Horch S., Chorkendorff I. and Nørskov J.K. J. Am. Chem. Soc., 127-15 (2005) 5308-5309.

[19] Lukowski M.A., Daniel A.S., Meng F., Forticaux A., Li L. and Jin S. Enhanced hydrogen evolution catalysis from chemically exfoliated metallic MoS2 nanosheets. J. Am. Chem. Soc., 135-28 (2013) 10274-10277.

[20] Li Y., Wang H., Xie L., Liang Y., Hong G. and Dai H. MoS2 nanoparticles grown on graphene: An advanced catalyst for the hydrogen evolution reaction. J. Am. Chem. Soc., 133-19 (2011) 7296-7299.

[21] Chorkendorff I., Bonde J., Jorgensen K.P., Horch S., Jaramillo T.F. and Nielsen J.H. Identification of Active Edge Sites for Electrochemical H2 Evolution from MoS2 Nanocatalysts. Science, 317-5834 (2007) 100102.

[22] Junfeng X., Hao Z., Shuang L., Ruoxing W., Xu S., Min Z., Jingfang Z., David L.X.W. and Yi X. Defect-Rich MoS2 Ultrathin Nanosheets with Additional Active Edge Sites for Enhanced Electrocatalytic Hydrogen Evolution. $A d v$. Mater., 25-40 (2013) 5807-5813.

[23] Li H., Duan X., Wu X., Zhuang X., Zhou H., Zhang Q., Zhu X., Pan A. and Duan X. Growth of Alloy MoS2xSe2(1-x) nanosheets with fully tunable chemical compositions and optical properties. J. Am. Chem. Soc., 136-10 (2014) 811.

[24] Nipane A., Karmakar D., Kaushik N., Karande S. and Lodha S. Few-Layer MoS2 p-Type Devices Enabled by Selective Doping Using Low Energy Phosphorus Implantation. ACS Nano., 10-2 (2016) 2128-2137.

[25] Yang L., Majumdar K., Liu H., Du Y., Wu H., Hatzistergos M., Hung P.Y., Tieckelmann R., Tsai W., Hobbs C. and Ye P.D. Chloride molecular doping technique on 2D materials: WS2 and MoS2. Nano Lett., 14-11 (2014) 62756280.

[26] Xu W., Huang Z., Zhu D., Xiong X., Lu Y., Chen L., He Z., Liu W., Liu Q., Ao J.P., Jiang H., Ang K.W., Han S., Yu W., Cao P., Wu J., He J. and Liu X. Monolayer WxMo1-xS2 Grown by Atmospheric Pressure Chemical Vapor Deposition: Bandgap Engineering and Field Effect Transistors. Adv. Funct. Mater., 27-13 (2017) 1606469.

[27] Zhang M., Wu J., Zhu Y., Dumcenco D.O., Hong J., Mao N., Deng S., Chen Y., Yang Y., Jin C., Chaki S.H., Huang Y.S., Zhang J., Xie L. Two-dimensional molybdenum tungsten diselenide alloys: Photoluminescence, Raman scattering, and electrical transport. ACS Nano, 8-7 (2014) 7130-7137.

[28] Li B., Huang L., Zhong M., Huo N., Li Y., Yang S., Fan C., Yang J., Hu W., Wei Z. and Li J. Synthesis and Transport Properties of LargeScale Alloy Co0.16Mo0.84S2 Bilayer Nanosheets. ACS Nano, 9-2 (2015) 1257-1262.

[29] Deng J., Li H., Xiao J., Tu Y., Deng D., Yang H., Tian H., Li J., Ren P. and Bao X. Triggering the electrocatalytic hydrogen evolution activity of the inert two-dimensional MoS2 surface via single-atom metal doping. Energy Environ. Sci., 8-5 (2015) 1594-1601.

[30] Gao G., Sun Q. and Du A. Activating catalytic inert basal plane of molybdenum disulfide to optimize hydrogen evolution activity via defect doping and strain engineering. J. Phys. Chem. C., 120-30 (2016) 16761-16766.

[31] Shi Y., Zhou Y., Yang D.R., Xu W.X., Wang C., Bin Wang F., Xu J.J., Xia X.H. and Chen H.Y. Energy Level Engineering of MoS2 by Transition-Metal Doping for Accelerating Hydrogen Evolution Reaction. J. Am. Chem. Soc., 139-43 (2017) 15479-15485.

[32] Wang H., Tsai C., Kong D., Chan K., AbildPedersen F., Nørskov J.K. and Cui Y. Transition-metal doped edge sites in vertically aligned MoS2catalysts for enhanced hydrogen evolution. Nano Res., 8-2 (2015) 566-575. 
[33] Zhang J., Feng J., Ma X., An C., Sun Y., Li J., Chi Y. and Liu J. Ultrathin Co(Ni)-doped MoS2 nanosheets as catalytic promoters enabling efficient solar hydrogen production. Nano Res., 9-8 (2016) 2284-2293.

[34] Ye R., Del Angel-Vicente P., Liu Y., ArellanoJimenez M.J., Peng Z., Wang T., Li Y., Yakobson B.I., Wei S.H., Yacaman M.J. and Tour J.M. High-Performance Hydrogen Evolution from MoS2(1-x)Px Solid Solution. Adv. Mater., 28-7 (2016) 1427-1432.

[35] Kiran V., Mukherjee D., Jenjeti R.N. and Sampath S. Active guests in the MoS2/MoSe2 host lattice: Efficient hydrogen evolution using few-layer alloys of MoS2(1-x)Se2x. Nanoscale, 6-21 (2014) 12856-12863.

[36] Sun X., Dai J., Guo Y., Wu C., Hu F., Zhao J., Zeng X. and Xie Y. Semimetallic molybdenum disulfide ultrathin nanosheets as an efficient electrocatalyst for hydrogen evolution. Nanoscale, 6-14 (2014) 8359-8367.

[37] Lewis D.J., Tedstone A.A., Zhong X.L., Lewis E.A., Rooney A., Savjani N., Brent J.R., Haigh S.J., Burke M.G., Muryn C.A., Raftery J.M., Warrens C., West K., Gaemers S. and O'Brien P. Thin films of molybdenum disulfide doped with chromium by aerosol-assisted chemical vapor deposition (AACVD). Chem. Mater., 274 (2015) 1367-1374.
[38] Yang L., Zhang J., Xiang B., Wang W. and Fu Q. MoS 2(1-x) Se 2x Nanobelts for Enhanced Hydrogen Evolution. Electrochim. Acta., 185 (2015) 236-241.

[39] Gershinsky G., Stern C., Shokhen V., Miroshnikov Y., Zitoun D., Gotlib N. and Naveh D. On the impact of Vertical Alignment of MoS2 for Efficient Lithium Storage. Sci. Rep., 7-1 (2017) 1-11.

[40] Li H., Zhang Q., Yap C.C.R., Tay B.K., Edwin T.H.T., Olivier A. and Baillargeat D. From bulk to monolayer MoS2: Evolution of Raman scattering. Adv. Funct. Mater., 22-7 (2012) 1385-1390.

[41] Chakraborty B., Matte H.S.S.R., Sood A.K. and Rao C.N.R. Layer-dependent resonant Raman scattering of a few layer MoS2. J. Raman Spectrosc., 44-1 (2013) 92-96.

[42] Zhang W., Huang J.K., Chen C.H., Chang Y.H., Cheng Y.J. and Li L.J. High-gain phototransistors based on a CVD MoS2 monolayer. Adv. Mater., 25-25 (2013) 34563461.

[43] Liu Y., Woods J.M., Shen J., Sun Y., Cha J.J. and Jung Y. Metal Seed Layer ThicknessInduced Transition From Vertical to Horizontal Growth of MoS2 and WS2. Nano Lett., 14-12 (2014) 6842-6849. 